\title{
Management of Type II Diabetes using Metformin- Loaded Microparticulate Gastroretentive Delivery Systems
}

\author{
Sara Salatin $^{1,2 *}$ and Mitra Jelvehgari ${ }^{1 *}$ \\ ${ }^{1}$ Department of Pharmaceutics, Tabriz University of Medical Sciences, Iran \\ ${ }^{2}$ Student Research Committee, Tabriz University of Medical Sciences, Iran
}

*Corresponding author: Sara Salatin, Mitra Jelvehgari, Department of Pharmaceutics, Faculty of Pharmacy, Tabriz University of Medical Sciences, Tabriz, Iran.

To Cite This Article: Sara Salatin, Management of Type II Diabetes using Metformin-Loaded Microparticulate Gastroretentive Delivery Systems. 2020 - 7(5). AJBSR.MS.ID.001195. DOI: 10.34297/AJBSR.2020.07.001195.

Received: 眥 February 10, 2020; Published: 眥 March 02, 2020

\begin{abstract}
Metformin is an oral anti-hyperglycemic derivative extensively used for the treatment of type II diabetes. Following oral administration, metformin exhibits an incomplete absorption and therefore repeated administrations of high doses of this drug are required for successful therapy due to its short biological half-life. For this, development of novel drug delivery systems for metformin might be useful to decrease the dosing frequency, to enhance its bioavailability, to reduce gastrointestinal adverse effects, and to be helpful for effective use of metformin in diabetes treatment. In this review, we present microparticulate gastroretentive delivery systems developed to enhance the oral bioavailability of metformin.
\end{abstract}

\section{Introduction}

The incidence of type II diabetes has dramatically increased throughout the world in recent years. Metformin is an oral antihyperglycemic agent widely used for lowering blood glucose concentrations in patients with type II diabetes [1,2]. It is a hydrophilic base which exists as the cationic species (>99.9\%) at physiological $\mathrm{pH}$. For this, passive diffusion of metformin across cell membranes is very limited. After oral administration, metformin is primarily absorbed from the upper small intestine and poses low bioavailability problems (its bioavailability is approximately 50$60 \%$ ). The biological half-life ( $\mathrm{t} 1 / 2$ ) of metformin is about $0.9-2.6 \mathrm{~h}$ [3]. Therefore, repeated administration of metformin with higher doses (500 or $850 \mathrm{mg}$ two to three times a day with or after meals) is required to achieve modest glucose-lowering effects. This may result in a higher incidence of gastrointestinal side-effects and can also lead to non-compliance. Therefore, an interest increased towards novel drug delivery systems (DDSs) that can remain in the stomach for an extended and predictable period of time. Recent studies reported that incomplete absorption of metformin can be improved using convenient microparticulate gastroretentive DDSs (MGDDSs) [4].

The terms "microparticle" refers to particles where the dimensions of the particle are measured in micrometers (typically $1 \mu \mathrm{m}$ to $1000 \mu \mathrm{m}$ ) and exhibit pronounced structural differences [5]. MGDDSs have high loading capacity and many polymers can be used such as gelatin, starch, hydroxypropyl cellulose, hydroxypropyl methylcellulose, polyacrylamine, polymethacrylate, and polyalkylcyanoacrylate. The microparticulate dosage forms are better localized in the gastrointestinal tract and are less likely to cause local irritation compared to the single unit systems [6]. Besides, these systems possess many advantages over conventional dosage forms such as site-control of drug delivery, low risk of dose dumping, effective protection of drugs against enzymatic degradation, increasing the bioavailability of drug, and also reduction of drug's side effects and repeated dosing [7]. Until now, various MGDDSs have been designed and developed, i.e. floating, mucoadhesive, high density (sinking), expandable, unfoldable, and 
super porous systems. The most extensively investigated types of MGDDSs include microtablets, micropellets, microgranules, microballons, microspheres, microcapsules etc.

Several papers are available in the literature in regard to MGDDSs for localizing the metformin to a particular region of gastrointestinal tract and to extend the gastric residence time [8] prepared cellulose acetate microspheres containing metformin for gastric retention and revealed that plasma glucose levels declined rapidly (at $30 \mathrm{~min}$ ). Similarly, Khonsari \& co-workers [9] reported that gastric-mucoadhesive microspheres loaded with metformin are a suitable system for prolongation of gastric residence time. Another work is involved in gastric-mucoadhesive microcapsules developed [10]. The results of this study revealed that microcapsules with controlled release properties and good mucoadhesive properties were prepared. Different polymers (eudragit RL 100 and cellulose acetate) was also used for preparation of sustained release metformin floating microcapsules [11].

\section{Conclusion}

In this short review, we aimed to update our understanding of metformin MGDDS. It may be concluded that MGDDSs offer various potential advantages for metformin with low bioavailability due to maximize its absorption and enhance absolute bioavailability.

\section{Acknowledgement}

The authors would like to acknowledge the support received from the Faculty of Pharmacy at Tabriz University of Medical Sciences (grant number. 63454)

\section{References}

1. Raza H, Javeria S, Rashid Z (2020) Sustained released Metformin microparticles for better management of Type II Diabetes Mellitus: Invitro studies. Mater Res Express In press.
2. Irons BK, Minze MG (2014) Drug treatment of type 2 diabetes mellitus in patients for whom metformin is contraindicated. Diabetes Metab Syndr Obes 7: 15-24.

3. Cetin M, Sahin S (2016) Microparticulate and nanoparticulate drug delivery systems for metformin hydrochloride. Drug deliv 23(8): 27962805.

4. Pandit V, Pai RS, Yadav V (2013) Pharmacokinetic and pharmacodynamic evaluation of floating microspheres of metformin hydrochloride. Drug Dev Ind Pharm 39(1): 117-127.

5. Salatin S (2018) Nanoparticles as potential tools for improved antioxidant enzyme delivery. J Adv Chem Pharm Mater (JACPM) 1(3): 65-66.

6. Javadzadeh Y, Hamedeyazdan S (2012) Novel drug delivery systems for modulation of gastrointestinal transit time. Rec Adv Novel Drug Carr Systems: Intech Open.

7. Mahmoudian M, Salatin S, Khosroushahi AY (2018) Natural low-and high-density lipoproteins as mighty bio-nanocarriers for anticancer drug delivery. Cancer Chemother Pharmacol 82(3): 371-382.

8. Choudhury PK, Kar M, Chauhan CS (2008) Cellulose acetate microspheres as floating depot systems to increase gastric retention of antidiabetic drug: formulation, characterization and in vitro-in vivo evaluation. Drug Dev Ind Pharm 34(4): 349-354.

9. Khonsari F, Zakeri Milani P, Jelvehgari M (2014) Formulation and Evaluation of In-vitro Characterization of Gastric-Mucoadhesive Microparticles/Discs Containing Metformin Hydrochloride. Iran J Pharm Res 13(1): 67-80.

10. Kumar A, Balakrishna T, Rajiv (2011) Formulation and evaluation of mucoadhesive microcapsules of metformin $\mathrm{HCl}$ with gum karaya. Int J Pharm Pharm Sci 3: 150-5.

11. Nath B, Nath LK, Mazumdar B (2009) Design and development of metformin $\mathrm{HCl}$ floating microcapsules using two polymers of different permeability characteristics. IJPSN. 